\title{
Tus derechos. Mis derechos. ¿Nuestros derechos?
}

\author{
Flavia Andrea NAVÉS1, Mariana THOMAS MORO2, Cecilia MOSCUZZA1, Gabriela BARONTINI1 \\ 1Universidad de Buenos Aires (Argentina)-CONCEBIR asociación civil. ${ }^{2}$ CONICET-CONCEBIR asociación civil (Argentina). \\ Autor para correspondencia: Flavia Andrea Navés. Correo electrónico: flaviaadreanaves@gmail.com \\ Recibido el 20 de julio de 2018; aceptado el 13 de septiembre de 2018.
}

Cómo citar este artículo: Navés FA, Thomas-Moro M, Moscuzza C, Barontini G. Tus derechos. Mis derechos. ¿Nuestros derechos?. Rev Med Cine [Internet] 2019;15(3): 153159.

DOI: http://dx.doi.org/10.14201/rmc2019153152159

\begin{abstract}
Resumen
Actualmente la sociedad argentina se debate entre la aceptación y el rechazo de la legalidad de la interrupción temprana del embarazo en un contexto socio-político de lucha por los derechos reproductivos y no reproductivos. Debate que suele perder de vista el padecimiento psíquico de las mujeres que deciden abortar.

La serie televisiva "Sexo en la ciudad" ("Sexo en Nueva York" en España)/ "Sex and the City" (1998-2004), creada por Darren Star y protagonizada por Sarah Jessica Parker, Kristin Davis, Cynthia Nixon y Kim Cattrall recrea con humor, ironía y dramatismo, pero, también con madurez y decisión temas socialmente controvertidos como el aborto y la infertilidad.

Elegimos el capítulo 11 de la temporada 4 titulado Podría, habría, debería (Podría haber, debería haber, si hubiera en España)/ Coulda, Woulda, Shoulda (2001) para realizar el presente trabajo. El objetivo es presentar, mediante un análisis situacional, el padecimiento subjetivo de las mujeres que recurren a la fertilización asistida, por un lado y de quienes deben tomar la decisión de interrumpir el embarazo, por el otro.
\end{abstract}

Palabras clave: embarazo; aborto; infertilidad.

\section{Your rights. My rights. Our rights?}

\begin{abstract}
Summary
Nowadays argentine society debates between the acceptance and rejection of the legality of the early interruption of pregnancy, in a socio-political context of struggle for reproductive and non-reproductive rights. Debate that usually lost sight of the mental suffering of women who decide an abortion.

The TV series "Sex and the City" (1998-2004), created by Darren Star and starring by Sarah Jessica Parker, Kristin Davis, Cynthia Nixon and Kim Cattrall recreates with humor, irony and drama, but also with maturity and decision, controversial social issues such as abortion and infertility.

We chose the chapter 11 of season 4 entitled Could, would, should (2001) to perform the actual work. The objective is to present, through a situational analysis, the subjective suffering of both sides, women who must draw on assisted fertilization technologies, on the one hand; and on the other, women who must make the decision to interrupt a pregnancy.
\end{abstract}

Keywords: pregnancy; abortion; infertility. 


\section{Ficha técnica}

Título en Argentina: "Sexo en la ciudad" (Serie de TV).

Título en España: "Sexo en Nueva York" (Serie de TV).

Título original: "Sex and the City" (TV Series).

País: Estados Unidos.

Año: 1998-2004.

Creador: Darren Star.

Dirección: Michael Patrick King, Sarah Jessica Parker.

Guión: Candace Bushnell, Darren Star, Michael Patrick King, Jenny Bicks, Cindy Chupack, Julie Rottenberg, Elisa Zuritsky, Allan Heinberg, Nicole Avril, Terri Minsky, Amy Harris. En principio la serie se basó en un recopilación de artículos de Candace Bushnell ("Sex and the City") publicados en el "New York Observer". Sin embargo, a partir de la segunda temporada, la serie empezó a volar por su cuenta. Más tarde originó la película homónima del año 2008 y su segunda parte en el año 2010.

Música: Bob Christianson, Douglas J. Cuomo, Didier Rachou, Kenneth Burgomaster, Alan Ari Lazar.

Intérpretes: Sarah Jessica Parker, Kim Cattrall, Kristin Davis, Cynthia Nixon.

Color: color.

Duración: 6 Temporadas con 94 capítulos de 30 minutos.

Género: comedia romántica.

Sinopsis: narra las aventuras amorosas y sexuales de cuatro neoyorquinas solteras e independientes: la columnista Carrie Bradshaw (Sarah Jessica Parker) y sus tres mejores amigas, la abogada Miranda (Cynthia Nixon), la pija Charlotte York (Kristin Davis) y la promiscua Samantha Jones (Kim Cattrall).

Premios: Ganó 7 Premios Emmy y 8 Globos de Oro. De 2000 a 2004.

Productora: Darren Star Productions, Home Box Office (HBO), Sex and the City Productions. Enlaces:

https://www.imdb.com/title/tt0159206

https://www.filmaffinity.com/ar/film721086.html

\section{Trailer en español}

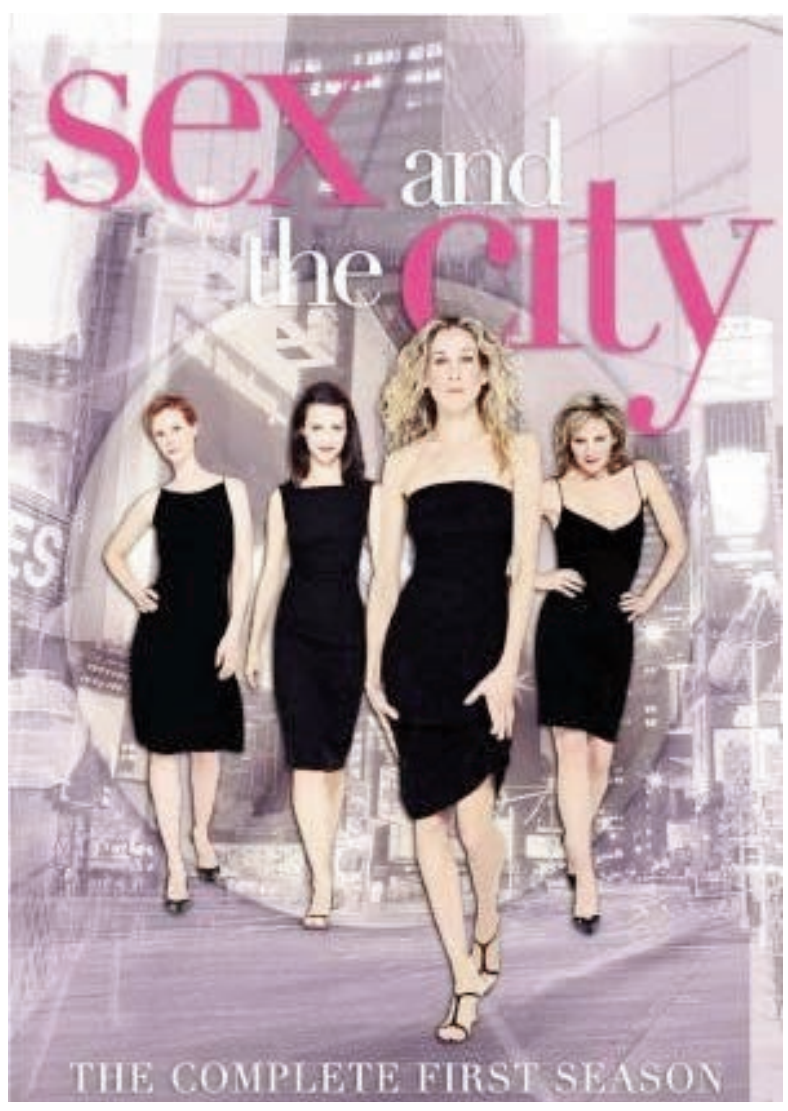

"Sexo en la ciudad," la serie

"Sex and the City" (1998-2004) creada por Darren Star es una serie televisiva estadounidense basada en el libro escrito por Candace Bushnell que lleva su nombre. En España se conoció con el nombre de Sexo en Nueva York y en hispanoamérica como Sexo en la ciudad. Protagonizada por Carrie Bradshaw (Sarah Jessica Parker), Charlotte York (Kristin Davis), Miranda Hobbes (Cynthia Nixon) y Samantha Jones (Kim Cattrall) tuvo una duración de 94 (noventa y cuatro) episodios distribuidos a lo largo de 6 (seis) temporadas compuestas por entre 12 y 20 capítulos cada uno.

Se trata de una serie ambientada en la ciudad de Nueva York que da vida a cuatro mujeres cosmopolitan de unos treinta y pico de años, independientes, universitarias, lograron insertarse profesionalmente, poseen una acomodada posición económica y viven solas.

Con humor, ironía y dramatismo la serie aborda problemas socialmente relevantes, como el papel de la mujer en la sociedad mostrando cómo, por fuera del ámbito del amor maternal y conyugal, las mujeres pueden ver ampliadas sus opciones accediendo a los bienes de 
consumo, al poder, al saber y al éxito profesional. Pero, también aborda con madurez y decisión temas socialmente controvertidos como el aborto, la infertilidad, el cáncer, la homosexualidad y las relaciones sexuales ocasionales a partir de las historias de vida de sus protagonistas.

En el trascurrir de las temporadas vemos cómo cada una de las protagonistas se relaciona con los afectos según su propio estilo. Miranda Hobbes es una abogada exitosa, siempre se ha centrado en su carrera, suele ser muy cínica a la hora de hablar de las relaciones y de los hombres. Sin embargo, se enamora de Steve, un camarero quien de ambición no sabe nada. En las primeras temporadas vemos como esta falta de ambición y la inestabilidad económica de él se convierte en un obstáculo para la pareja. Charlotte York, muy tradicional y conservadora, es corredora de arte. Ella sueña con encontrar a su "príncipe azul"; a lo largo de las temporadas vemos que logra casarse con Trey MacDougal un sofisticado millonario y cirujano. Con quién se divorciará ya que a pesar de provenir de "una buena familia" y de cumplir a la perfección con sus exigencias parece ser impotente. La separación dura un breve tiempo ya que Trey logra vencer los obstáculos que le impedían relacionarse sexualmente con ella, pero, los avances de él ponen al descubierto la imposibilidad de la pareja de lograr un embarazo. Finalmente sus desacuerdos acerca de si deben o no buscar la fertilización in vitro los llevan al divorcio. Carrie Bradshaw escribe una columna de frecuencia semanal para el periódico "The New York Star". Enamorada de Mr. Big, un hombre que nunca termina de comprometerse con ella, luchará por su amor hasta las últimas consecuencias. Samantha Jones es la de mayor edad del grupo y trabaja en relaciones públicas. Cree que ha tenido muchas almas gemelas e insiste en que sus compañeros sexuales se tienen que ir después de que ella haya llegado al clímax. En la cuarta decide «hacerse» lesbiana, al integrar a su vida con María. Luego comienza una relación con su jefe, Richard, el magnate hotelero de quien se separa al encontrarlo con otra mujer. En la sexta temporada, le diagnostican cáncer de mama cuando decide hacerse los chequeos necesarios para realizarse una cirugía estética; vence exitosamente al cáncer luego de varias sesiones de quimioterapia y gracias al apoyo incondicional de Smith Jerrod, un modelo publicitario 20 años menor que ella con el que comenzó teniendo sexo casual y de quién termina enamorada.

\section{La entrada en situación}

El capítulo 11 (once) de la temporada 4 (cuatro) titulado "Podría, habría, debería" da entrada a la controversia que rodea el ámbito de la salud sexual, reproductiva y no reproductiva.
Inicia el capítulo con la noticia de que Miranda está embaraza. Ella decide compartir la noticia con su mejor amiga Carrie; ese día, minutos más tarde, compartirían el almuerzo semanal con el resto de sus amigas.

Carrie pregunta, entre la sorpresa y el espanto: “¿Estás embarazada? ¿En serio? ¿Estás segura?”

La ironía de Miranda no se hace esperar -"No... sólo creí que sería divertido decirlo" responde. Acto seguido le explica: -"Me hice tres pruebas de embarazo y vengo de ver a mi doctor. iPor eso te llamé para que me veas aquí en la esquina de la 23 si me muero! iDormí con Steve! Una vez, en singular. Un sexo piadoso porque sólo tiene un testículo... iParece que el otro era sólo ornamental! iDios! iSoy una perfecta idiota! iPor qué no use un condón! iEl sólo tiene un testículo y yo sólo tengo un ovario flojo! ¿En qué clase de mundo crea eso a un bebé?"

Mientras Miranda y Carrie hablaban... Charlotte recibía una desagradable sorpresa: "Su período"

¿Cómo contarle a Charlotte que estoy embarazada se pregunta Miranda? Ella sabe que su amiga sueña con tener un bebé. Un bebé que a ella le "vino de arriba" que no programó, que no desea...

Finalmente llega el momento de confrontar su realidad con la realidad de una de sus mejores amigas en el almuerzo semanal que las reúne. Charlotte es la última en llegar con sus tampones en la mano como muestra activa de su frustración. Al mismo tiempo que se sienta a la mesa exclama: -"iJamás me embarazaré! Cuando pienso en tantas mujeres cada día embarazándose, embarazándose, iembarazándose... lo he intentado cinco meses y nada! iUna mujer de 35 tiene el 30\% de posibilidades de concebir y las cifras disminuyen desde ahí!!"

La mirada despreocupada de Samantha no se hace esperar y se refleja en sus palabras: - ¿Si están difícil embarazarse cómo explicas el número de niños en los aviones?" Despertando las risas de Miranda y Carrie, pero, al mismo tiempo el enojo de Charlotte quién exclama: - "Esto no es cuestión de risas...Es por mí... tal
vez no puedo tener bebés. Trey resultó estar
bien. Es por mí, sé que es por mí. Siempre oigo
historias de personas que se embarazaron el
uno al otro. Yo lo he hecho de cabeza, de lado,
de espaldas, lo he hecho arriba, abajo, abajo y
arriba y luego abajo. ¿Saben cuántas veces
Trey y yo lo hemos hecho sin condón? i73!
¿Tienen idea de cuánto semen perfectamente 
bueno es eso? Suficiente para hacerme saber en el fondo que soy yo. $Y$ lo peor es que temí desde los 20 que me embarazaría... podría haber tenido sexo con todo lo que viera. Debería haber tenido la suerte de embarazarme por error..."

Miranda y Carrie cruzan miradas durante toda la conversación y Charlotte parece inadvertirlo, sin embargo, no tarda en preguntar:

-“¿Qué son esa miradas Miranda? Estás sentada ahí, no has dicho una palabra y miras solo a Carrie... ¿Me crees patética Miranda?”

Miranda se siente entre la espada y la pared. Pero, sabe que debe abrir el juego y exponer su realidad.

M: -"No se me ocurre un peor momento para decir esto... exclama"

C: -"mmm por dios..."

Ch: -"i¿Oigan, qué carajos pasa?!"

M: - "Me embarace..."

Ch: - Ah..."

M: -"Steve..."

S: - ¿Sexo piadoso? Ah...”

Ch: -"iÉl sólo tiene una bola no es justo!"

C: - "Charlotte, respira. Ten, toma agua."

Ch: -“¿Cómo pudiste hacerme esto?”

$\mathrm{M}:$ - ¿¿Ati?”

C: -"Muy bien basta, por favor. Basta"

S: -¿¿Qué harás?”

Miradas

S: - "Síl.. ok"

Ch: - ¿Ni siquiera vas a considerar conservarlo?”

M: - "Esto no está en mis planes ahora...y la verdad es que me siento mal hablando de ello frente a ti..."
Ch: - "Pues me iré"

M: - "No..."

Ch: -"Para que se queden aquí y tengan su abortiva charla"

C: -"Vamos Charlotte, por favor. Siéntate. Y mirándola a Miranda dice: -No hablaba enserio... lo único que puede pensar ahora son bebés..."

M: - "Me hizo sonar tan..."

S: -"iNo lo digas! iDe acuerdo no es una agradable situación pero pasa! Todas la hemos pasado alguna vez..."

\section{M: - " YYo nunca! Exclama horrorizada"}

S: - Yo tuve dos. ¿Carrie, cuantos tuviste?"

C: - "Ah... uno"

S: - "Ay, cierto... ese mesero"

C: -"iPor favor no lo vuelvas peor de lo que fue... "él era mesero en Desalum! ¿Entiendes? iDéjame recordarte que en 1988 era muy popular!"

M: -"Parecía serlo..."

\section{Mis derechos, tus derechos}

El diálogo entre las protagonistas refleja a las claras el padecimiento subjetivo de cada una. Padecimiento que parece quedar subsumido en las discusiones morales sobre lo que es correcto o incorrecto en el ámbito de la salud sexual reproductiva y no reproductiva.

La culpa, la moral, los prejuicios sociales se ponen en juego en el presente diálogo. ¿Qué hace que Charlotte reaccione de ese modo? ¿Por qué Miranda siente culpa ante la mirada prejuiciosa de su amiga?

Miranda a diferencia de Charlotte elige tomar la decisión de interrumpir el embarazo al que accedió por azar ya que nunca formó parte de su deseo ser madre o tener un hijo. Esta decisión, en Argentina, aún está penada por la Ley y, por lo tanto, se encuentra contemplada en el Código Penal Argentino, promulgado en el Siglo XIX más precisamente en el año 1886. En él puede leerse los artículos 85, 86, 87 y 88 del "Libro Segundo. De los 
Delitos". "Título I. Delitos contra las personas", Capítulo I de "Delitos contra la vida" hacen mención al aborto. Los artículos 85 y 87 establecen la pena para quienes lo causaren. El artículo 88 penaliza a la mujer que cause su propio aborto o autorizase a un tercero para realizarlo. Sólo el artículo № 86 que se refiere al aborto profesional y a las figuras impunes: abortos terapéuticos, eugenésicos y sentimental -producida la gestación por violación- sufrió cuatro modificaciones, la última corresponde al año 1984 que reimpone la redacción original del Código Penal de 1922.

Luego de más de cuarenta años de espera para que el aborto legal, gratuito y seguro sea legalizado en Argentina, entre los meses de abril y junio del año 2018, forma parte de la agenda política y fue tratado en reuniones informativas convocadas por las Comisiones de Legislación General, Legislación Penal, Acción Social y Salud Pública, y Familia, Mujer, Niñez y Adolescencia de la Cámara de Diputados de la Nación con el fin de que se traten los diversos proyectos de ley vinculados con el aborto y presentados en la Cámara de Diputados de la Nación Argentina. Finalmente, el 14 de junio del mismo año obtuvo media sanción en la Cámara de Diputados de la Nación Argentina el Proyecto de Ley de aborto -Expte Senado CD/22/18- cuyo objetivo es garantizar el derecho de acceder a la interrupción voluntaria del embarazo con el solo requerimiento de la mujer o persona gestante hasta la semana catorce, inclusive, del proceso gestacional; y fuera de ese plazo en los siguientes casos:

1. Si el embarazo fuera producto de una violación, con el solo requerimiento y la declaración jurada de la mujer o persona gestante ante el/la profesional de la salud interviniente;

2. Si estuviera en peligro la vida o la salud de la mujer o persona gestante, considerada como derecho humano;

3. Si se diagnosticara la inviabilidad de vida extrauterina del feto.

Este proyecto de Ley se encuentra a la espera del incipiente debate que tendrá lugar en la Cámara de Senadores de la Nación Argentina.

¿Cuál es el eje que guía el debate actual en Argentina sobre un tema que atañe a la decisión de una mujer que, como Miranda, no contempla la posibilidad de tener hijos y busca subsanar su error mediante la interrupción temprana del embarazo?
En los años '70 se argumentaba a favor del aborto poniendo el énfasis en el derecho de la mujer a decidir sobre su propio cuerpo. Una década más tarde según el Centro de Bioética, Persona y Familia1 "la expansión de la ecografía permitió el acceso a la vida intrauterina y la humanidad del niño por nacer se hizo visible. Eso puso en evidencia que estábamos ante un nuevo ser humano que nos exigía respeto y cuidado, de modo que la necesidad de garantizar su derecho a la vida modificó los ejes del debate. Si ya nos encontramos ante un ser humano, no podemos admitir que se le quite la vida sin quebrar las bases mismas de la convivencia social” (pp. 2). Finalmente, un nuevo cambio de perspectiva tuvo lugar en los años '90 y, en lugar de hacer foco en el derecho de la mujer a decidir sobre su propio cuerpo, se pone el acento en la desigualdad de las condiciones de acceso al aborto entre las mujeres que pueden pagarlo y aquellas que no tienen la posibilidad de hacerlo en condiciones de higiene que garanticen su vida. Reduciendo el debate a la pugna del derecho a la vida de la mujer vs. el derecho a la vida del niño por nacer.

El capítulo 11 de la cuarta temporada de Sexo en la Ciudad manifiesta la puja entre los derechos de Miranda a decidir sobre su propio cuerpo accediendo a la interrupción temprana del embarazo y los derechos de Charlotte de conformar una familia ya sea de forma natural, por adopción o por técnicas de reproducción asistida tal como lo contempla el Código Civil y Comercial Argentino desde el año 2015. Esta puja de derechos parece reflejar, por un lado, los argumentos de los años '70 a favor del aborto legal y, por el otro, introduce la discusión sobre el inicio de la vida reduciendo la querella sobre el aborto legal, gratuito y seguro a la tensión entre la vida y la muerte de una potencial persona.

\section{Subjetividad vs derechos}

Así planteada la discusión, en el plano de los derechos reproductivos y no reproductivos entre Miranda y Charlotte ¿Qué hay de la subjetividad puesta en juego en cada decisión?

Si la cultura le otorga identidad y valoración social a la mujer mediante su acceso a la maternidad ¿Qué lugar para el deseo de una mujer que, como Miranda, no pretende ser madre?

En Estados Unidos el aborto es legal, sin embargo su legalidad no impide el sufrimiento de Miranda. Ella se siente mal, se siente culpable por haberse descuidado. Su decisión tiene un costo y no es sólo la relación con su amiga. ¿Puede un aborto terminar con su desliz? ¿Puede 
ella sola tomar la decisión de interrumpir su embarazo? ¿Qué decisión tomará Steve? Son algunos de los interrogantes que invaden la mente de Miranda.

La escena nos muestra como Samantha intenta aflojar la tensión que se produjo por la reacción de Charlotte y sin más que la absoluta sinceridad exclama:

-iDe acuerdo no es una agradable situación pero pasa! Todas la hemos pasado alguna vez.... Comentario que estimula la respuesta horrorizada de Miranda: -iYo nunca! Exclamación que nos advierte de la culpa que emerge en Miranda.

¿Taponará la culpa la emergencia subjetiva en Miranda obturando toda posibilidad de decidir? No lo sabemos.

¿Es acaso genuino el dolor de Charlotte y, en cambio, para Miranda sólo está reservada la culpa de su error?

Charlotte, a diferencia de Miranda, es infértil. Es decir que padece una enfermedad definida por la Organización Mundial de la Salud (OMS) como una enfermedad del sistema reproductivo, como la incapacidad de lograr un embarazo clínico después de 12 meses o más de relaciones sexuales no protegidas (para mujeres de menos a 35 años) o de seis meses para mujeres mayores a 35 años.

Desde el punto de vista psicológico, Antequera Jurado, Moreno Rosset, Jenaro y Ávila Espada 2 plantean que la infertilidad puede considerarse como una enfermedad crónica que provoca un gran impacto emocional en quienes la padecen, comparable a la encontrada en pacientes oncológicos.

Charlotte nos muestra sus alteraciones emocionales, cognitivas y comportamentales producto de las múltiples pérdidas de embarazos o deseos no concretados y duelos no elaborados. Ella pierde el control al ver que su amiga se embaraza sin más esfuerzo que una noche de "sexo piadoso" con un hombre que sólo tiene un testículo y su amiga con jun solo ovario! ¿Por qué todos se embarazan menos yo? Es la pregunta que resuena en la escena del restaurante en el que las amigas comparten uno de sus almuerzos semanales.

Cuando sobreviene una crisis como la que atraviesa Charlotte todos los recursos y herramientas conocidas se cuestionan, deben plantearse nuevas reglas. La flexibilidad, la capacidad de adaptación y el aprendizaje de herramientas que ayuden en el manejo de la incertidumbre y la frustración, resulta fundamental.

No solo se pierde la fertilidad, sino muchas veces la posibilidad de concebir hijos biológicos, o genéticamente vinculados, también se pierde el sentido de la sexualidad que termina siendo examinada y controlada por un tercero, se pasa del placer a la obligación intentando "acertar" justo con el día fértil, se desdibuja y se cuestiona el proyecto de familia, se desvanecen los sueños de crianza, se ven alterados los planes que se tenían como proyecto de vida.

Explica Thomas Moro que en Infertilidad se habla de la montaña rusa emocional, ya que los estados emocionales fluctúan y se alternan, de forma espontánea y repentina durante los diferentes momentos del proceso, desde el diagnostico hasta el inicio y cierre de cada tratamiento ${ }^{3}$.

Los encuentros sexuales entre Charlotte y Trey se limitan a la búsqueda de las posturas adecuadas para quedar embaraza, ya no hay deseo en el encuentro, sólo la necesidad de conseguir un embarazo. La obsesión de Charlotte por lograr el embarazo pone en jaque su relación con Trey. Ella quiere tener un hijo a cualquier costo, aunque ese implique recurrir a la medicina reproductiva; él, en cambio, no está dispuesto aunque la ama.

Todo aquello que se creía que se podía lograr de una forma simple y rápida, y que solo había que tomar la decisión, no sucede así. La infertilidad cuestiona y socava la autoestima y autoimagen de los individuos que la padecen, su valor y posición frente al mundo (frente a su pareja, a su familia de origen, frente a sus amigos que quizá ya lograron ser padres, en las relaciones laborales y la comunidad en general), se desvanece la capacidad de control.

Charlotte nos enseña que la ansiedad por la falta de control y los pensamientos acerca del futuro se alternan con momentos de tristeza y depresión por la falta de esperanza y la sensación de que nunca se podrá lograr un embarazo. Por momentos la ira y el enojo la invaden, decide aislarse y alejarse de su entorno, de sus amigas, porque se siente incomprendida; adopta una posición negadora e intolerante y también siente culpa por no haber iniciado antes la búsqueda. La envidia y los celos que le despertó Miranda quien quedó embaraza fácilmente y hasta sin quererlo la invaden hasta resultarle intolerable escuchar el dolor de su amiga y sin más se levanta y se va ofendida. 


\section{A modo de cierre: ¿nuestros derechos?}

En el campo de la salud sexual, reproductiva y no reproductiva queda mucho por hacer, las barreras psicosociales y religiosas obturan el desarrollo genuino de normativas que legitimen las prácticas médicas a las que la mujer se expone.

Mientras que el aborto no sea legitimado y los grupos de poder sigan centrando la tensión en términos de estar o no a favor de la vida del potencial niño por nacer los vacíos legales seguirán haciendo estragos en la subjetividad de quienes necesitan hacer valer sus derechos reproductivos y no reproductivos.

Tal como atestigua Navés acceder a la interrupción temprana de un embarazo sin los cuidados necesarios ni las mejores condiciones de higiene es en muchos casos sinónimo de empujar a las mujeres más pobres a una futura infertilidad, a sentir que su cuerpo está incompleto por no poder procrear cuando realmente lo desea y muchas veces a quedar en estado de abandono porque la hombría, en esta cultura, todavía se mide en hijos ${ }^{4}$.

Las mujeres reportan a la infertilidad como el evento más perturbador de sus vidas y experimentan mayores niveles de estrés que los hombres, asociado también a los diagnósticos, los tratamientos físicos y farmacológicamente invasivos; a su vez asumen la responsabilidad, son las que buscan información y ayuda; buscan adaptarse y entender buscando el diálogo y expresando sus sentimientos. Pero, también suelen abandonar el sueño de tener un bebé dado el gran estrés emocional que provoca hacer frente a la dificultad para concebir ya que el dolor que genera esta imposibilidad suele provocar, a demás, aislamiento familiar, hastío y agobio y, así, alejarse del proyecto parental suele resultar la mejor salida de esta situación ${ }^{5}$.

En los hombres concebir un hijo es prueba de su masculinidad. Culturalmente el sexo y la fertilidad están intrínsecamente ligados y resulta difícil separar la capacidad de un hombre de concebir niños, de su virilidad. En contraposición con las mujeres, los hombres utilizan estilos que incorporan la negación, y la evitación, les cuesta pedir ayuda, no suelen compartir sus sentimientos y se ubican como soporte de sus mujeres.

\section{Referencias}

1. Centro de Bioética, Persona y Familia. Análisis del proyecto de ley de aborto libre y propuestas para la maternidad vulnerable [Internet]. 2018.

2. Antequera Jurado R, Moreno-Rosset C, Jenaro C, Ávila Espada, A. Principales trastornos psicológicos asociados a la infertilidad. Papeles del Psicólogo 2008; 29(2): 167-75.
3. Thomas Moro M. La montaña rusa emocional. En Saliendo del laberinto. Cien Lunas, Buenos Aires; 2016.

4. Navés, F. (2017). Aborto y tecnologías reproductivas: ¿Derechos reproductivos para el cuerpo femenino?. Eticar [Internet].

5. Barontini G. Mitos (ajenos y propios) sobre (in) fertilidad. En Saliendo del laberinto. Cien Lunas, Buenos Aires; 2016

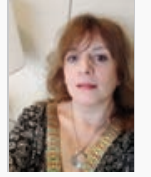

Flavia Andrea Navés es Licenciada en Psicología de la Universidad de Buenos Aires. Maestranda y especialista en Tecnología educativa, UBA. Psicóloga Orientada en Reproducción Humana y Fertilización Asistida de la Sociedad Argentina de Medicina Reproductiva (SAMeR). Docente de la Práctica Profesional. Investigadora en Proyectos UBACYT. Integrante e investigadora del equipo de psicología y musicoterapia de CONCEBIR Asociación Civil. Integrante e investigadora del Capítulo de Psicología de SAMeR.

Mariana Thomas Moro es licenciada en Psicología Clínica (UB) con posgrado en Psicología Perinatal y de la Primera Infancia (UBA-CIIPME-CONICET). Posee una formación intensiva en Psicología de la Infertilidad (ALMER) y de la Reproducción Humana (Red LARA). Es Psicóloga orientada en reproducción humana y fertilización asistida (SAMeR), miembro del Capítulo de Psicología de SAMeR y integrante e investigadora del Equipo de Psicología y Musicoterapia de CONCEBIR Asociación Civil.

Cecilia Andrea Moscuzza es licenciada en Musicoterapia con diploma de honor de la Facultad de Psicología (UBA). Es miembro del equipo de Psicología y Musicoterapia de CONCEBIR Asociación Civil, integrante del equipo de Investigación de Psicología y Musicoterapia de CONCEBIR Asociación Civil, miembro de la Asociación Argentina de Musicoterapia y del Comité Editorial de la Revista Ecos. Ha publicado artículos académicos y de investigación sobre infertilidad y musicoterapia. 